\title{
A binary engine fuelling HD 87643's complex circumstellar environment
}

\section{Determined using AMBER/VLTI imaging ${ }^{\star, \star \star}$}

\author{
F. Millour ${ }^{1}$, O. Chesneau ${ }^{2}$, M. Borges Fernandes ${ }^{2}$, A. Meilland ${ }^{1}$, G. Mars ${ }^{3}$, C. Benoist ${ }^{3}$, E. Thiébaut ${ }^{4}$, P. Stee $^{2}$, \\ K.-H. Hofmann ${ }^{1}$, F. Baron ${ }^{5}$, J. Young ${ }^{5}$, P. Bendjoya ${ }^{2}$, A. Carciofi ${ }^{6}$, A. Domiciano de Souza ${ }^{2}$, T. Driebe ${ }^{1}$, S. Jankov ${ }^{2}$, \\ P. Kervella ${ }^{7}$, R. G. Petrov ${ }^{2}$, S. Robbe-Dubois ${ }^{2}$, F. Vakili ${ }^{2}$, L. B. F. M. Waters ${ }^{8}$, and G. Weigelt ${ }^{1}$
}

\author{
1 Max-Planck Institut für Radioastronomie, Auf dem Hügel 69, 53121 Bonn, Germany \\ e-mail: FMillour@mpifr-bonn.mpg.de \\ 2 UMR 6525 H. Fizeau, Univ. Nice Sophia Antipolis, CNRS, Observatoire de la Côte d'Azur, 06108 Nice Cedex 2, France \\ 3 UMR 6202 Cassiopée, Univ. Nice Sophia Antipolis, CNRS, Observatoire de la Côte d'Azur, BP 4229, 06304 Nice, France \\ 4 UMR 5574 CRAL, Univ. Lyon 1, Obs. Lyon, CNRS, 9 avenue Charles André, 69561 Saint Genis Laval Cedex, France \\ 5 Astrophysics Group, Cavendish Laboratory, University of Cambridge, J.J. Thomson Avenue, Cambridge CB3 OHE, UK \\ ${ }^{6}$ Instituto de Astronomia, Geofísica e Ciências Atmosféricas, Universidade de São Paulo, Rua do Matão 1226, Cidade Universitária, \\ 05508-900, São Paulo, SP, Brazil \\ 7 LESIA, Observatoire de Paris, CNRS UMR 8109, UPMC, Univ. Paris Diderot, 5 place Jules Janssen, 92195 Meudon Cedex, France \\ 8 Faculty of Science, Astronomical Institute "Anton Pannekoek", Kruislaan 403, 1098 SJ Amsterdam, The Netherlands
}

Received 24 December 2008 / Accepted 2 August 2009

\begin{abstract}
Context. The star HD 87643, exhibiting the "B[e] phenomenon", has one of the most extreme infrared excesses for this object class. It harbours a large amount of both hot and cold dust, and is surrounded by an extended reflection nebula.

Aims. One of our major goals was to investigate the presence of a companion in HD87643. In addition, the presence of close dusty material was tested through a combination of multi-wavelength high spatial resolution observations.

Methods. We observed HD 87643 with high spatial resolution techniques, using the near-IR AMBER/VLTI interferometer with baselines ranging from $60 \mathrm{~m}$ to $130 \mathrm{~m}$ and the mid-IR MIDI/VLTI interferometer with baselines ranging from $25 \mathrm{~m}$ to $65 \mathrm{~m}$. These observations are complemented by NACO/VLT adaptive-optics-corrected images in the $K$ and $L$-bands, and ESO-2.2m optical WideField Imager large-scale images in the $B, V$ and $R$-bands.

Results. We report the direct detection of a companion to HD 87643 by means of image synthesis using the AMBER/VLTI instrument. The presence of the companion is confirmed by the MIDI and NACO data, although with a lower confidence. The companion is separated by $\sim 34$ mas with a roughly north-south orientation. The period must be large (several tens of years) and hence the orbital parameters are not determined yet. Binarity with high eccentricity might be the key to interpreting the extreme characteristics of this system, namely a dusty circumstellar envelope around the primary, a compact dust nebulosity around the binary system and a complex extended nebula suggesting past violent ejections.
\end{abstract}

Key words. techniques: high angular resolution - techniques: interferometric - stars: emission-line, Be - binaries: close circumstellar matter - stars: individual: HD 87643

\section{Introduction}

Stars with the "B[e] phenomenon" are B-type stars with strong Balmer emission lines, numerous permitted Fe II lines, and forbidden $\mathrm{O} I$ and $\mathrm{Fe}$ II lines in their optical spectrum. In addition, these stars exhibit a strong near and mid-IR excess due to circumstellar dust (Allen \& Swings 1976; Conti 1997). For brevity, we henceforth refer to stars showing the $\mathrm{B}[\mathrm{e}]$ phenomenon as "B[e] stars". The class of $\mathrm{B}[\mathrm{e}]$ stars is composed of several subclasses, including objects at different evolutionary stages and of low, medium, and high mass (Lamers et al. 1998). Distances

\footnotetext{
* Based on observations made with the ESO very large telescope at Paranal Observatory under programs 076.D-0575, 077.D-0095, 076.D0141, 380.D-0340, and 280.C-5071, with the ESO 1.52-m and archival ESO data.

$\star \star$ Appendix is only available in electronic form at

http://www. aanda.org
}

toward them are usually poorly known. As a consequence, the determination of the evolutionary state of $\mathrm{B}[\mathrm{e}]$ stars is often uncertain.

Observations suggest that the wind of some of the most massive $\mathrm{B}[\mathrm{e}]$ stars (the so-called supergiant $\mathrm{B}[\mathrm{e}]$ stars or $\mathrm{sgB}[\mathrm{e}]$ in Lamers et al. 1998) is composed of two distinct components, as proposed by Zickgraf et al. (1985). The first component is a wind of low density in the polar regions; the second component is a wind of high density and low velocity located in the equatorial region of the star. The thermal infrared excess is supposed to be produced in the outer parts of the equatorial wind where the temperature allows the formation of dust grains, but dust might also survive much closer to the star in a dense and compact disc.

The formation of dusty environments around $\mathrm{B}[\mathrm{e}]$ evolved objects is a challenge, with many theoretical issues (see Kraus \& Lamers 2003). A non-spherical, disc-like environment may be 
the key to understanding dust formation in $\mathrm{sgB}[\mathrm{e}]$ stars. It could be attributed to the rapid rotation of these stars (e.g. Lamers \& Pauldrach 1991; Bjorkman \& Cassinelli 1993; Kraus 2006; Zsargó et al. 2008) but could also be caused by companions, since some $\mathrm{B}[\mathrm{e}]$ stars were found to be binaries. Indeed, the number of companions detected around these objects is steadily growing (Miroshnichenko et al. 2004, 2006), but claims that all $\mathrm{B}[\mathrm{e}]$ stars are binaries are still controversial (Zickgraf 2003).

The number of $\mathrm{B}[\mathrm{e}]$ stars is rather limited, and many of them are unclassified (cited as unclB[e] in Lamers et al. 1998) because they exhibit properties usually associated with young and evolved objects simultaneously.

Our group has undertaken a large observing campaign to investigate these poorly studied objects ( $\operatorname{sgB}[\mathrm{e}]$ and un$\mathrm{clB}[\mathrm{e}])$ mostly using optical interferometry with the Very Large Telescope Interferometer (hereafter VLTI, see Stee et al. 2005, for an introduction to interferometric observations of $\mathrm{Be}$ and $\mathrm{B}[\mathrm{e}]$ stars). Early results were published on the sgB[e] star CPD572874 (Domiciano de Souza et al. 2007).

HD 87643 (Hen 3-365, MWC 198, IRAS 10028-5825) appears to be a special case among $\operatorname{sgB}[\mathrm{e}]$ and unclB[e] stars. It is a B2[e] star (Oudmaijer et al. 1998) that exhibits the largest infrared excess among this class and appears to be embedded in a complex nebula whose properties are reminiscent of the nebulae around LBVs (van den Bergh 1972; Surdej et al. 1981; Surdej \& Swings 1983). HD 87643 is considered a unclB[e] by Lamers et al. (1998): it was classified as $\operatorname{sgB}[\mathrm{e}]$ (see the estimation of the bolometric luminosity in McGregor et al. 1988; Cidale et al. 2001), under the assumption that it lies close to the Carina arm (2-2.5 kpc Oudmaijer et al. 1998; Miroshnichenko 1998; McGregor et al. 1988), but one can also find it classified as a Herbig star (Valenti et al. 2000; Baines et al. 2006). The $v \sin i$ of the central star is unknown since no pure photospheric line is detected in the visible spectrum. Clues to the disc-like geometry of the environment are provided by the polarisation ellipse in the U-Q plane across $\mathrm{H} \alpha$ (Oudmaijer et al. 1998). The detected PA is about $15^{\circ}-20^{\circ}$ (Yudin \& Evans 1998; Oudmaijer et al. 1998). On the other hand, Baines et al. (2006) claimed to have detected an asymmetric outflow around HD87643 using spectro-astrometry. However, they also note that "HD 87643 stands out in the complexity of its spectro-astrometry", compared with the numerous other Herbig Be stars they observed.

HD 87643 has displayed a long-term decline of the visual brightness for the last $30 \mathrm{yr}$, from $V \approx 8.5 / 8.8$ in 1980 (Miroshnichenko 1998), to $V \approx 9.3 / 9.4$ in early 2009 (Pojmański 2009). This decline is superimposed on shorter-term variations, such as a 0.5 mag decrease in $\approx 1$ month, followed by a 0.7 mag increase in $\approx 5$ months, seen in the ASAS photometric survey (Pojmański 2009) between JD 2453000 and JD 2453180. Yudin \& Evans (1998) also noted that HD87643 shows Algollike variability, the star showing variations "on a timescale of days", and being "bluer during brightness minima".

The totality of the ISO/SWS $2-45 \mu \mathrm{m}$ mid-IR emission from HD 87643 seems concentrated within the smallest ISO aperture $\left(14^{\prime \prime} \times 22^{\prime \prime}\right)$, and at $10 \mu \mathrm{m}$, the dusty environment is unresolved on a 1" scale (Voors 1999). The ISO/LWS spectrum also continues from the shorter-wavelength SED without any jump, which is an additional sign that the mid-IR emission is compact. The disc-like geometry is strongly supported by the huge infrared excess exhibited by the source, the large absorption of the central star flux, and the polarimetric data. Voors (1999) proposed that the disc might be a circumbinary disc.

In this paper we report new observations that bring a new insight into this interesting object, proving the presence of a
Table 1. AMBER and MIDI observing logs.

\begin{tabular}{|c|c|c|c|}
\hline \multirow[b]{2}{*}{ Date } & \multirow[b]{2}{*}{ Stations } & \multicolumn{2}{|c|}{ Projected baseline } \\
\hline & & $\begin{array}{c}\text { Length } \\
{[\mathrm{m}]}\end{array}$ & $\begin{array}{c}\text { PA } \\
\text { [degrees] }\end{array}$ \\
\hline \multicolumn{4}{|c|}{ AMBER 1 (UT) } \\
\hline $18 / 02 / 2006,3 \mathrm{~h}$ & UT1-3-4 & $95,57,129$ & $22,88,46$ \\
\hline $18 / 02 / 2006,8 \mathrm{~h}$ & UT1-3-4 & $71,62,102$ & $65,145,102$ \\
\hline \multicolumn{4}{|c|}{ AMBER 2 (AT) } \\
\hline $01 / 03 / 2008,2 \mathrm{~h}$ & $\mathrm{~K} 0-\mathrm{G} 1-\mathrm{A} 0$ & $83,78,128$ & $-168,-94,-132$ \\
\hline $05 / 03 / 2008,3 \mathrm{~h}$ & G1-D0-H0 & $63,64,60.0$ & $-59,64,3$ \\
\hline $06 / 03 / 2008,5 \mathrm{~h}$ & G1-D0-H0 & $69,60,58$ & $-37,90,19$ \\
\hline $06 / 03 / 2008,7 \mathrm{~h}$ & G1-D0-H0 & $71,52,33$ & $-15,117,33$ \\
\hline $10 / 03 / 2008,3 \mathrm{~h}$ & H0-G0-E0 & $31,15.6,46.8$ & -102 \\
\hline $11 / 03 / 2008,0 \mathrm{~h}$ & H0-G0-E0 & $32,16,47$ & -147 \\
\hline $12 / 03 / 2008,0 \mathrm{~h}$ & H0-G0-E0 & $32,16,48$ & -145 \\
\hline \multicolumn{4}{|c|}{ MIDI (AT) } \\
\hline $26 / 02 / 2006,4 \mathrm{~h}$ & D0-G0 & 31.3 & 76.3 \\
\hline $27 / 02 / 2006,6 \mathrm{~h}$ & A0-G0 & 57.1 & 101.3 \\
\hline $01 / 03 / 2006,1 \mathrm{~h}$ & A0-G0 & 63.5 & 38.1 \\
\hline $01 / 03 / 2006,5 \mathrm{~h}$ & A0-G0 & 61.1 & 85.1 \\
\hline $19 / 04 / 2006,4 h$ & D0-G0 & 25.4 & 123.1 \\
\hline $23 / 05 / 2006,2 \mathrm{~h}$ & A0-G0 & 51.7 & 120.0 \\
\hline $25 / 05 / 2006,1 \mathrm{~h}$ & A0-G0 & 54.4 & 110.6 \\
\hline
\end{tabular}

companion, a resolved circum-primary dust envelope (most likely a disc), and circumbinary material.

The outline of the article is as follows: the observations and data recorded are presented in Sect. 1, then we present and discuss the main results from our observing campaign in Sect. 2, and give a global view of the system, considering this new information, in Sect. 3.

\section{Observations and data processing}

\subsection{AMBER/VLTI near-IR interferometry}

HD 87643 was observed at the ESO/Paranal observatory with the Astronomical Multi BEam Recombiner (AMBER), the nearinfrared instrument of the VLTI (Petrov et al. 2007). The observations were carried out on February 18, 2006 in medium spectral resolution $(R=1500)$ and during a series of nights in March 2008 at low spectral resolution $(R=35)$. AMBER uses three $8 \mathrm{~m}$ telescopes (Unit Telescopes, hereafter UT) or three $1.8 \mathrm{~m}$ telescopes (Auxiliary Telescopes, hereafter AT). The calibration stars used were HD 109787, HD 86440, HD 101531, HD 63744 and $\epsilon$ Oph. Details of the observations can be found in Table 1.

The data were processed with the standard AMBER data reduction software (amdlib 2.1, see for instance Tatulli et al. 2007; Millour et al. 2004) plus a series of advanced scripts to calibrate the data (Millour et al. 2008). The AMBER DRS performs a fringe fitting instead of Fourier transforms and computes interferometric data products such as $V^{2}$ and closure phases (for a review of the interferometric data analysis, see: Haniff 2006; Monnier 2006; Millour 2008). We performed the reduction using standard selection criteria (see for instance Appendix $\mathrm{C}$ in Millour et al. 2007) for the individual exposures, rejecting $80 \%$ of the data before averaging the data products. The additional scripts allowed us to compute realistic error bars, including the uncertainties on the diameters of the calibration stars, the instrument atmosphere transfer function instabilities, and the fundamental noise. The study of the transfer function provided by different calibrators gives an estimate of absolute errors on $V^{2}$ 
between 0.05 and 0.1 . The visibilities and closure phases are shown in Fig. 3.

\subsection{MIDI/VLTI mid-IR interferometry}

The observations of HD 87643 at the ESO/Paranal observatory using the MID-Infrared instrument (hereafter MIDI, Leinert et al. 2003, 2004) were carried out from February until May 2006. MIDI is the mid-infrared ( $N$-band, 7.5-13.5 $\mu \mathrm{m})$ twotelescope combiner of the VLTI, operating like a classical Michelson interferometer. In our case, only the ATs were used since HD 87643 is bright in the mid-IR (156 Jy). We used a standard MIDI observing sequence, as described by Ratzka et al. (2007). The source was observed in the so-called High-Sens mode, implying that the photometry from each individual telescope is performed subsequently to the recording of the fringes. The low spectral resolution $(R=30)$ provided by the prism was used. The data consist of 7 visibility spectra and one flux spectrum (PSF 1.2" with ATs). The errors on the visibilities, including the internal ones and those from the calibrator diameter uncertainty, range from 0.05 to 0.15 . The MIDI spectrum was difficult to calibrate, and the accuracy of the absolute photometry is not better than $30 \%$, although one can see in Fig. 4 that the MIDI spectrum agrees well with the IRAS (Olnon et al. 1986) and the ISO spectra (affected by problems of "gluing" between different spectral bands, as reported in Voors 1999). The log of the observations is given in Table 1. We used two different MIDI data reduction packages: MIA developed at the Max-Planck-Institut für Astronomie and EWS developed at the Leiden Observatory (MIA+EWS ${ }^{1}$, ver.1.5.1). The resulting visibilities can be seen in Fig. 4.

\subsection{NACO/VLT adaptive optics assisted imaging}

We observed HD 87643 at the ESO/Paranal observatory with the NACO adaptive optics camera (NAos adaptive optics system combined with the COnica camera, Rousset et al. 2003) attached to UT4 of the Very Large Telescope (VLT). NACO was operated in the visual wavefront sensor configuration. We observed the target with $K \mathrm{~s}(2.2 \mu \mathrm{m})$ and $L^{\prime}(3.8 \mu \mathrm{m})$ broad-band filters. The star HD 296986 was used to derive the point-spread function (PSF). The S13 camera mode was used for Ks, with a 13 mas per pixel scale and a $14^{\prime \prime} \times 14^{\prime \prime}$ field of view. In $L^{\prime}$, using camera mode L27, the field of view was 28 " $\times 28$ " and the pixel scale was 27.1 mas. The auto-jitter mode was used, which, at each exposure, moves the telescope in a random pattern within a box of side $7^{\prime \prime}$ in Ks and $15^{\prime \prime}$ in $L^{\prime}$. The journal of observations can be found in Table 2 .

The data reduction was performed using our own scripts. First, bad pixels were removed (i.e. interpolated using the adjacent pixel values) and a flat-field correction was applied to the data. Then, the sky was computed as the median of all exposures and subtracted exposure by exposure. A visual inspection of each exposure allowed us to check the PSF quality. The "bad" ones were removed for the next step. This allowed us to significantly improve the final data product image quality compared to the standard pipeline-reduced frames. This also led us to disregard the 20/03/2008 data-sets, which were in any case flagged as "failed" in the observing log. A cross-correlation technique was then used to re-centre the images with about 1 pixel accuracy. Finally, all the selected frames were co-added, resulting in the total exposure time shown in Table 2.

\footnotetext{
1 Available at http://www.strw. leidenuniv.nl/ nevec/MIDI
}

Table 2. Journal of observations with NACO/VLT.

\begin{tabular}{lccccc}
\hline \hline Date & Star & $\begin{array}{c}\text { Used } \\
\text { filt. }\end{array}$ & $\begin{array}{c}\text { Used } \\
\text { cam. }\end{array}$ & $\begin{array}{c}\text { Exp } \\
\text { time }\end{array}$ & Seeing \\
\hline 20/03/2008, 6h22 & HD 87643 & $K_{\mathrm{s}}$ & S13 & $96 \mathrm{~s}$ & $1.13^{\prime \prime}$ \\
20/03/2008, 6h36 & HD296986 & $K_{\mathrm{s}}$ & S13 & $96 \mathrm{~s}$ & $1.10^{\prime \prime}$ \\
20/03/2008, 6h55 & HD 87643 & $L^{\prime}$ & L27 & $280 \mathrm{~s}^{*}$ & $1.48^{\prime \prime}$ \\
20/03/2008, 7h21 & HD296986 & $L^{\prime}$ & L27 & $324 \mathrm{~s}$ & $1.03^{\prime \prime}$ \\
21/03/2008, 3h33 & HD 87643 & $K_{\mathrm{s}}$ & S13 & $96 \mathrm{~s}$ & $0.93^{\prime \prime}$ \\
$21 / 03 / 2008,3 \mathrm{~h} 37$ & HD 87643 & $K_{\mathrm{s}}$ & S13 & $96 \mathrm{~s}$ & $0.90^{\prime \prime}$ \\
21/03/2008, 3h43 & HD 87643 & $K_{\mathrm{s}}$ & S13 & $84 \mathrm{~s}$ & $0.91^{\prime \prime}$ \\
21/03/2008, 3h57 & HD296986 & $K_{\mathrm{s}}$ & S13 & $96 \mathrm{~s}$ & $0.63^{\prime \prime}$ \\
$21 / 03 / 2008,4 \mathrm{~h} 21$ & HD 87643 & $L^{\prime}$ & L27 & $315 \mathrm{~s}$ & $1.14^{\prime \prime}$ \\
$21 / 03 / 2008,4 \mathrm{~h} 44$ & HD296986 & $L^{\prime}$ & L27 & $270 \mathrm{~s}$ & $1.73^{\prime \prime}$ \\
\hline
\end{tabular}

All stars were observed with a neutral density filter; ${ }^{*}$ saturated.

As a result, we got a pair of science star/PSF star images for each observation (which were repeated due to changing weather conditions and saturation of the detector). The PSF FWHM is $75 \pm 4$ mas in the $K_{\mathrm{s}}$-band and $111 \pm 6$ mas in the $L^{\prime}$-band. In addition to a deconvolution attempt presented in Sect. 2.2, we computed radial profiles to increase the dynamic range from about $\approx 10^{3}$ per pixel to $\approx 10^{4}-10^{5}$. The result can be found in Fig. 5.

\section{4. $2.2 \mathrm{~m} / \mathrm{WFI}$ large-field imaging}

We retrieved unpublished archival ESO/wide-field imager (WFI) observations of the nebula around HD 87643 from the ESO database $^{2}$ carried out on March 15 and 16, 2001. The WFI is a mosaic camera attached to the ESO $2.2 \mathrm{~m}$ telescope at the La Silla observatory. It consists of eight $2 \mathrm{k} \times 4 \mathrm{k}$ CCDs, forming an $8 \mathrm{k} \times 8 \mathrm{k}$ array with a pixel scale of $0.238^{\prime \prime}$ per pixel. Hence, a single WFI pointing covers a sky area of about $30^{\prime} \times 30^{\prime}$. The observations were performed in the $B, V, R_{\mathrm{c}}$ and $I_{\mathrm{c}}$ broadband filters and in the narrow-band filter in the $\mathrm{H} \alpha$ line $(\lambda=658 \mathrm{~nm}$, $F W H M=7.4 \mathrm{~nm}$ ). However, we used only the observations with $B, V$, and $R$ filters given that it is a reflection nebula only (Surdej et al. 1981). In order to cover the gaps between the WFI CCDs and to correct for moving objects and cosmic ray hits, for each pointing, a sequence of five offset exposures was performed in each filter.

The data reduction was performed using the package alambic developed by Vandame based on tools available from the multi-resolution visual model package (MVM) by Rué \& Bijaoui (1997). The image is shown in Fig. 6.

\section{New facts about HD 87643}

\subsection{Interferometry data: A binary star plus a compact dusty disc}

From the sparse 2006 AMBER medium spectral resolution data, the structure of the source could not be inferred unambiguously. The squared visibilities from 2006 did not show any significant variation with spatial frequency. The closure phases were measured as zero within the error estimates (see Fig. 3).

The Br $\gamma$ emission line appears clearly in the AMBER calibrated spectrum after using the technique described in Hanson et al. (1996) of fitting and removing the Br $\gamma$ line for the calibrator. The resulting $\mathrm{Br} \gamma$ line accounts for $15 \%$ of the

\footnotetext{
${ }^{2}$ http://archive.eso.org
} 


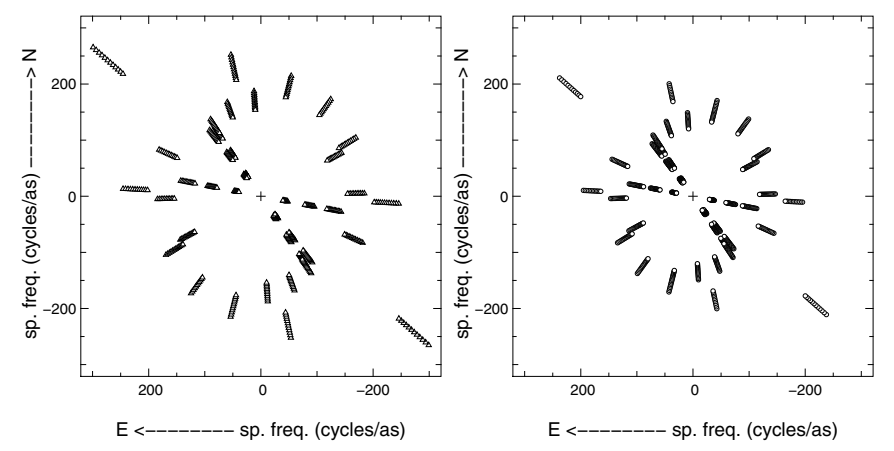

Fig. 1. UV coverage obtained on HD87643 in the $H$-band (left), in the $K$-band (right). The radial lines are caused by the different wavelengths within the bands.

continuum flux at its maximum and is spread over 5 spectral pixels $\left(250 \mathrm{~km} \mathrm{~s}^{-1}\right)$, similar to what is reported in McGregor et al. (1988).

The extensive AMBER low spectral resolution data, secured in 2008 (see Fig. 3), show large variations of $V^{2}$ and closure phases and clearly indicate a modulation from a binary source. Given the good $u v$ coverage (see Fig. 1) and the quality of the data, we undertook an image reconstruction process, aiming to determine the best models to use for the interpretation.

\subsubsection{Image reconstruction}

The images in Fig. 2 were reconstructed from the AMBER squared visibilities and closure phases using the MIRA software (Thiébaut 2008). MIRA compares the visibilities and the closure phases from a modelled image with the observed data using a cost-estimate optimisation, including a priori information such as image positivity (all image pixels are positive) and compactness of the source (using a so-called $L 2-L 1$ regularisation, see Thiébaut 2008, for details).

Separate image reconstructions were made using the $H$-band and $K$-band data, assuming that the object was achromatic over the whole band considered. MIRA needs a starting image, but we found that the image reconstruction result did not significantly depend on it. Extensive tests and comparisons with other software (Hofmann \& Weigelt 1993; Baron \& Young 2008, see Appendix A) made us confident that we could distinguish between artifacts and real structures in the images. The theoretical field of view, computed using the formula $2.44 \lambda / B_{\min }$, is $\approx 70$ mas, $B_{\min }$ being the shortest projected baseline $(15 \mathrm{~m})$. We convolved the $K$-band image with a Gaussian beam of $F W H M \lambda / B_{\max }=3.5$ mas and the $H$-band one with a beam of $\lambda / B_{\max }=2.7$ mas, $B_{\max }$ being the longest projected baseline $(128 \mathrm{~m})$. The image noise is of the order of $1 \%$.

The reconstructed image reveals the presence of a companion star with a plane-of-sky separation of $\approx 34.5$ mas (see Table 3 ). the $K$-band image also reveals an extended structure detected around the main star. We cannot assess any elongation of this resolved structure due to the poor UV coverage in the NW direction. We emphasise that there is still a $180^{\circ}$ uncertainty due to the overall uncertainty on the AMBER closure phase sign (and not due to the image reconstruction process: see Appendix A.1.3). We will refer in the following to "the northern" and "the southern" components.

We also extracted relative fluxes for the different components within the dashed circles in Fig. 2 (see Table 3), but one has to bear in mind that extracting such fluxes is affected not only by large errors, but also potentially by large systematics. Therefore, we also performed the flux measurements using model-fitting.

\subsubsection{Model fitting}

In Sect. 2.1.1, we show that the 2008 AMBER data can be directly interpreted assuming the presence of a binary source with a plane-of-sky separation of $\approx 34$ mas and a PA of $\approx 0^{\circ}$ (see Table 3) and a resolved southern component. We fitted the data using such a model to obtain more accurate component fluxes. The images show a series of large-scale artifacts (i.e. flux whose spatial location is poorly constrained), accounting for a large fraction of the total integrated flux. These artifacts are related to the lack of data with high visibilities at small spatial frequencies. We therefore also included an extended component to our model, fully resolved on all AMBER baselines.

Our model-fitting used the scientific software yorick ${ }^{3}$ combined with the AMBER data reduction software amdlib. This tool was complemented with a series of optimisation scripts developed by the JMMC (Béchet et al. 2005) and others developed by us. We judge that the resulting fits were satisfactory, even though the formal $\chi^{2}$ is about 40 (see Fig. 3), probably due to an underestimation of the errors during the data reduction.

The MIDI data show complex, spectrally dependent visibility variations from one observation to another (see Fig. 4). Spherical and even 2-D axi-symmetric dusty models were not able to account for this data set. We used the separation and P.A. inferred from the AMBER 2008 data as initial values for the fitting of the AMBER 2006 and MIDI 2006 measurements.

The result of such a fit shows that our binary model is compatible with both the AMBER and MIDI 2006 data sets. Moreover, we find that the MIDI separation is close to that derived from the AMBER 2008 data set. The P.A. of the secondary component differs slightly between near- and midinfrared, which might be due to an offset of the dust structure compared to the main star. The total flux seen by MIDI is dominated by a fully resolved background in addition to the unresolved binary components (Table 3). The AMBER 2006 data set is too limited to provide a strong constraint on the the flux ratios; therefore, we do not use them in the following analysis.

We stress that the MIDI data is affected by an independent $180^{\circ}$ orientation ambiguity to the AMBER ambiguity (due to the uncalibrated closure phase sign), as it only consists of squared visibility data. Hence, we cannot definitely relate the component fluxes derived from the MIDI data to those derived from the AMBER data.

\subsection{NACO K-band imaging: a very compact dusty environment}

We performed a deconvolution process on our NACO images. Using both speckle techniques and the Lucy-Richardson deconvolution algorithm, an elongated north-south compact structure could be detected in the $K$-band but not in the $L$-band. This is compatible with the AMBER data.

In addition, we tested the presence of dusty emission in the surroundings of the star by azimuthally integrating the flux to increase the dynamic range (see Fig. 5). In the $K$-band, no extended emission is detectable up to $1.5 \operatorname{arcsec}$ (at larger distances, ghost reflections or electronic ghosts impair the dynamic

\footnotetext{
3 Open-source scientific software freely available at the following URL: http://yorick. sourceforge.net
} 

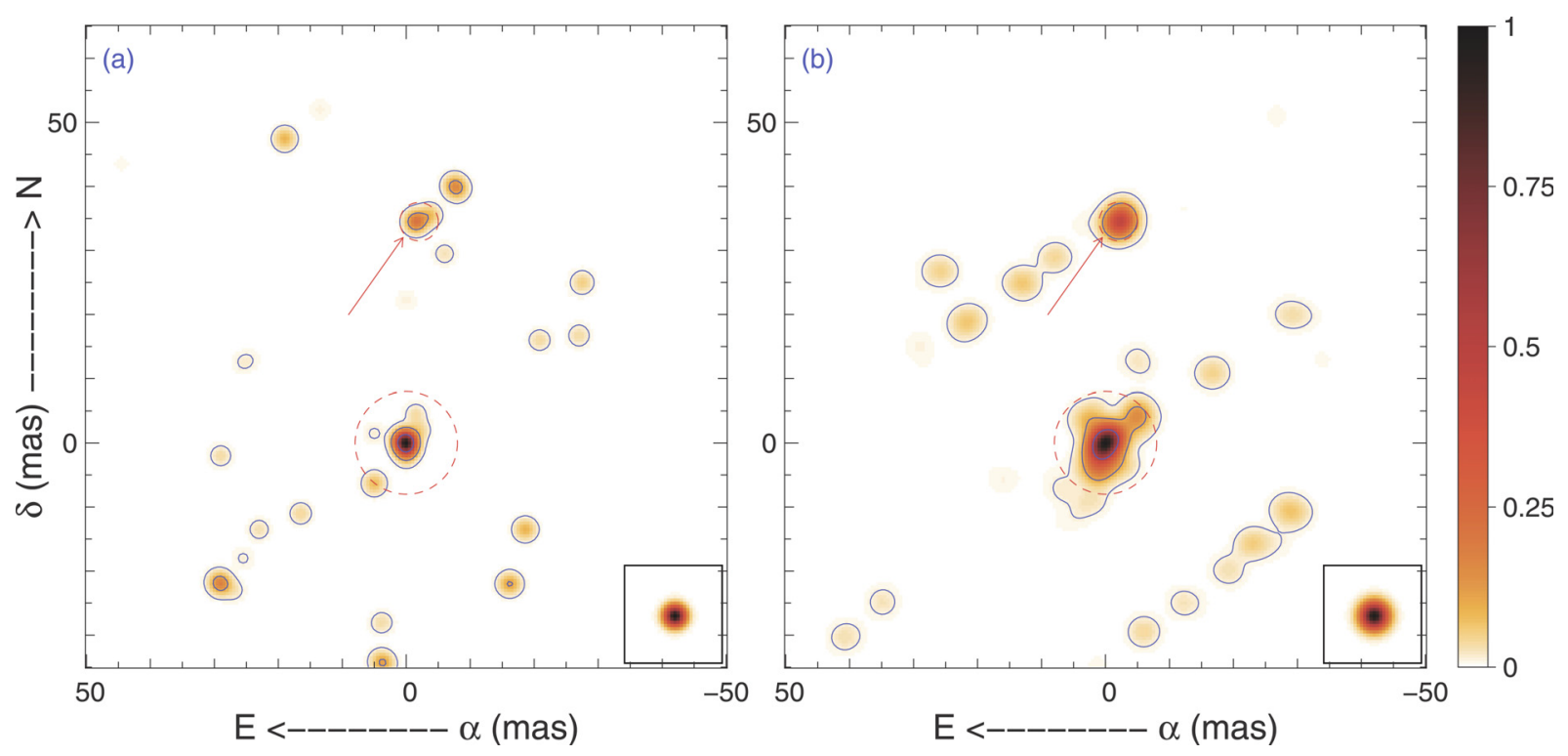

Fig. 2. Aperture-synthesis images of HD87643 reconstructed from the $H$-band AMBER data (panel 2a) and the $K$-band data (panel $2 b$ ), assuming the source is achromatic in each band. Contours with 50,10, and $1 \%$ of the maximum flux are shown, and the beam size is shown in the lower-right box. The arrow shows the companion star, whereas the dashed circles show the trusted structures in the images.

Table 3. Estimated diameter of the envelope, position of the secondary source, and respective fluxes, relative to the total flux.

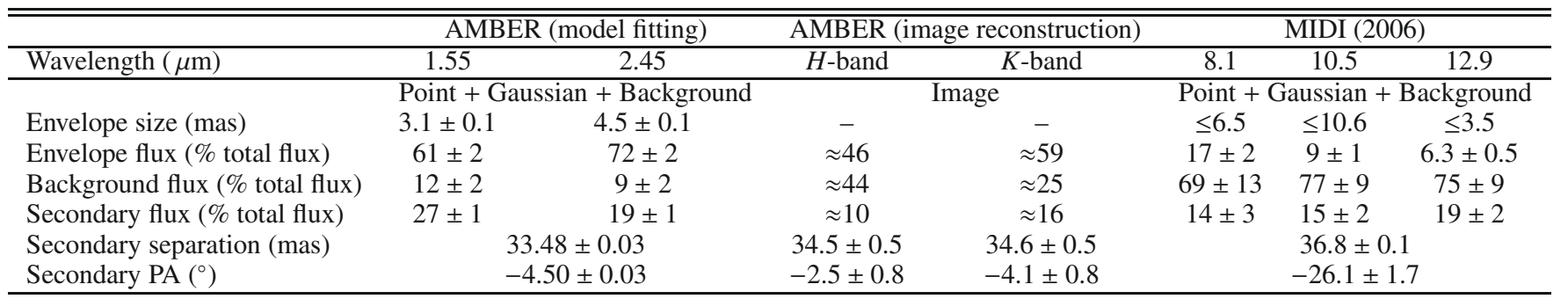

The envelope flux includes both the southern component and its compact circumstellar envelope fluxes. The errors given for the model-fitting reflects only formal estimates from the $\chi^{2}$ value.

range of the images) and a faint emission can be seen in the $L$ band from 0.5 to 3.0 arcsec. This might be linked to the fully resolved component seen in our AMBER data, and therefore probably implies that it is much more extended than the field of view of AMBER. However, the dynamic range of our images $\left(\approx 10^{3}\right.$ per pixel, and $\approx 10^{4}-10^{5}$ for the radial profiles $)$ does not allow us to access the spatial distribution of such a dusty nebula.

\subsection{WFI imaging: the large scale nebula revisited}

The extended nebula was discovered and studied by van den Bergh (1972); Surdej et al. (1981); Surdej \& Swings (1983). In particular, using the $3.6 \mathrm{~m}$ ESO telescope at La Silla, Surdej \& Swings (1983) showed that the structure was a reflection nebula with a $60-100^{\prime \prime}$ extension.

The nebula revealed by the WFI instrument (see Fig. 6) with the $2.2 \mathrm{~m}$ telescope at La Silla shows increased dynamic range but shares the same spatial resolution and global morphology as the previous works. The nebular structures are primarily seen in the north-west quadrant from the central star in the form of an extended and structured filamentary feature at distances of 15$20^{\prime \prime}$ (north) to $35-50^{\prime \prime}$ (west). In some places, labelled (B1) to (B4), the nebula appears blown-up by the central star wind, with clumpy and patchy features.
This structure has no extended counterpart in the south-east region, where arc-like structures (labeled (A1) to (A4) in the figure) can be seen closer to the star (at 10", 12", 14", and 18" respectively). These arcs are spaced by two to four arc-secs and might come from past ejection events.

In the south-west region, at $12^{\prime \prime}, 19^{\prime \prime}$, and $30^{\prime \prime}$ respectively from the star, two bright and one faint arc can be seen (labelled (A5) to (A7) in the figure). These arcs do not appear to be connected to the previously-mentioned ones.

A star count made using sextractor (Bertin \& Arnouts 1996) gives values twice as low in the east half than in the west half of the image. No significant relative change in the counts as a function of filter is detectable over the field. This indicates that there is a total absorption screen somewhere in the field, totally masking the background stars in the east half of the WFI image. We note that it corresponds to a dark cloud in the eastern part, clearly seen in our WFI image in front of red (hydrogen?) nebular emission. However, we cannot directly assess if this screen is in front or behind HD 87643 and its nebula.

\section{Discussion}

\subsection{Adopting a distance for the system}

HD 87643 is thought to be an evolved $\mathrm{B}[\mathrm{e}]$ star, and the nebula suggests a link to the LBV class (Surdej \& Swings 1983). 

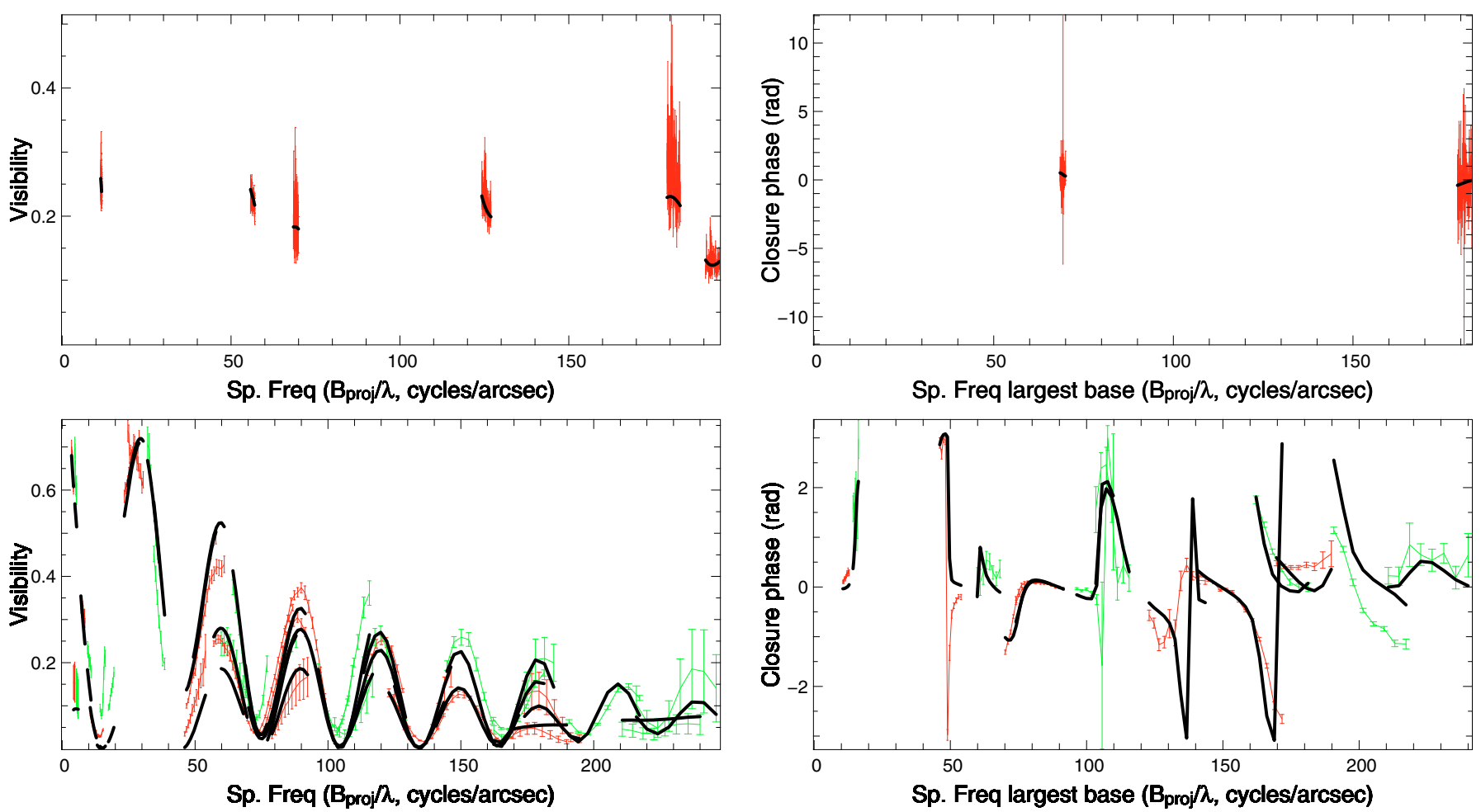

Fig. 3. Squared visibilities (left) and closure phases (right) plotted as a function of spatial frequency, projected onto the inferred binary position angle. The 2006 AMBER data set is seen on top, 2008 on the bottom. The model involving a binary system composed of one resolved and one unresolved component plus a fully resolved background is shown with a solid black line.

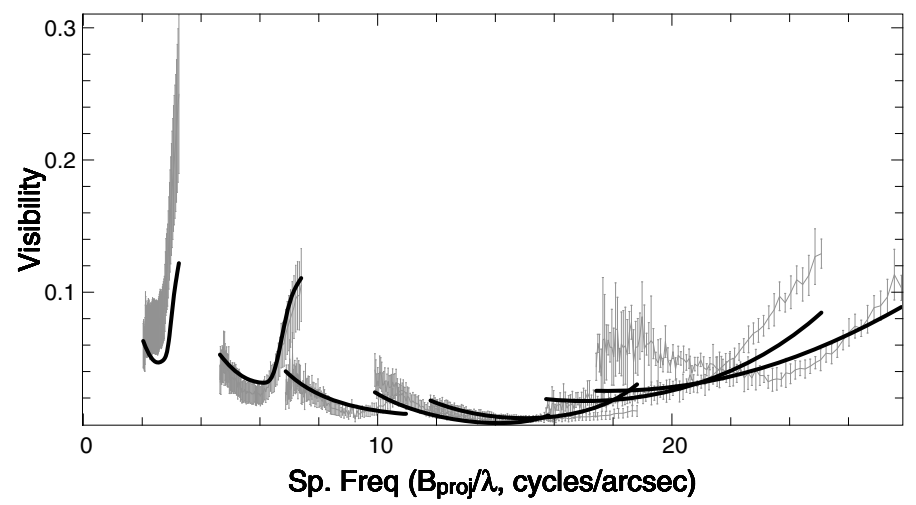

Fig. 4. MIDI visibilities (in Gray with error bars), projected on the direction of the detected binary star and compared with our model (in thick black line).

HD 87643 stands out as unusual compared to typical sgB[e] stars such as CPD-57 2874, since ISO SWS and LWS spectra show a considerable amount of cold dust $(T \leq 150 \mathrm{~K})$.

McGregor et al. (1988) implicitly assumed that HD 87643 is a supergiant in order to determine its distance, based on its location in the direction of the Carina Arm. Such a location would place it at a distance of 2-3 kpc. However, the distance of HD 87643 has never been accurately measured. When other estimates of the distance are found in the literature, the values are usually much smaller. These were derived by:

1. van den Bergh (1972) and de Freitas Pacheco et al. (1982): $530 \mathrm{pc}$, based on Kurucz models for a B2V star $\left(T_{\mathrm{eff}}=\right.$ $\left.18000 \mathrm{~K}, E(B-V)=0.63 \mathrm{mag}, R_{*}=6 R_{\odot}\right)$;
2. Surdej et al. (1981): $1 \mathrm{kpc}$, based on $M_{V}=-4.1$ derived from photometry;

3. Shore et al. (1990): $1.2 \mathrm{kpc}$, using the IUE UV spectrum;

4. Lopes et al. (1992): 2-3 kpc using the equivalent width (hereafter, EW) of NaI lines. We note, however, that the high reported value of $2.9 \mathrm{kpc}$ is affected by a factor of 2 error in their application of the statistical relation cited by Allen (1973). Considering the correct relation $(r=2.0 D, r$ being the distance in $\mathrm{kpc}$ and $\mathrm{D}$ being the mean EW in $\AA$ of the two Na D lines), the distance would be $1.46 \mathrm{kpc}$ (Lopes, private communication). The EW were measured using lowresolution spectra, implying a possible contamination of circumstellar origin;

5. Oudmaijer et al. (1998): 1-6 kpc, also based on the NaI Dline equivalent widths derived from a higher-resolution spectrum, but using data on the interstellar extinction for nearby stars instead of the relationship from Allen (1973). The large uncertainty of this distance is due to the large scatter of the extinction values in the Carina arm line of sight.

6. Zorec (1998): $1.45 \mathrm{kpc}$, based on a SED fitting using various assumptions.

Therefore, we arbitrarily use the distance of $1.5 \mathrm{kpc}$, as suggested by some of these works, in the subsequent parts of the present article.

\subsection{Orbital characteristics of the binary system}

One of the main results from the AMBER interferometric observations is that HD 87643 is a double system. In the $K$-band, the NACO image core is also significantly resolved with an elongation in the same direction as the AMBER binary. The separation and position angle of the components are well constrained by the 

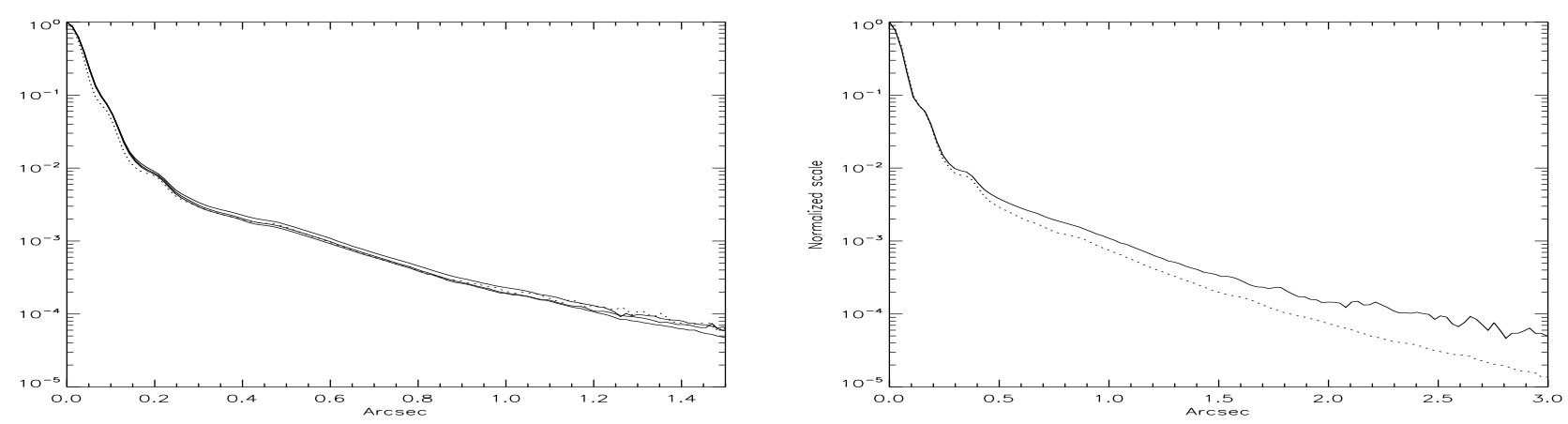

Fig. 5. NACO radial profiles. Left is the $K_{\mathrm{s}}$ filter and right is the $L^{\prime}$ filter. HD 87643 is shown as full lines ( 3 observations in $K$ s, and one in $L^{\prime}$, see Table 2). The calibrator star is shown as a dotted line.
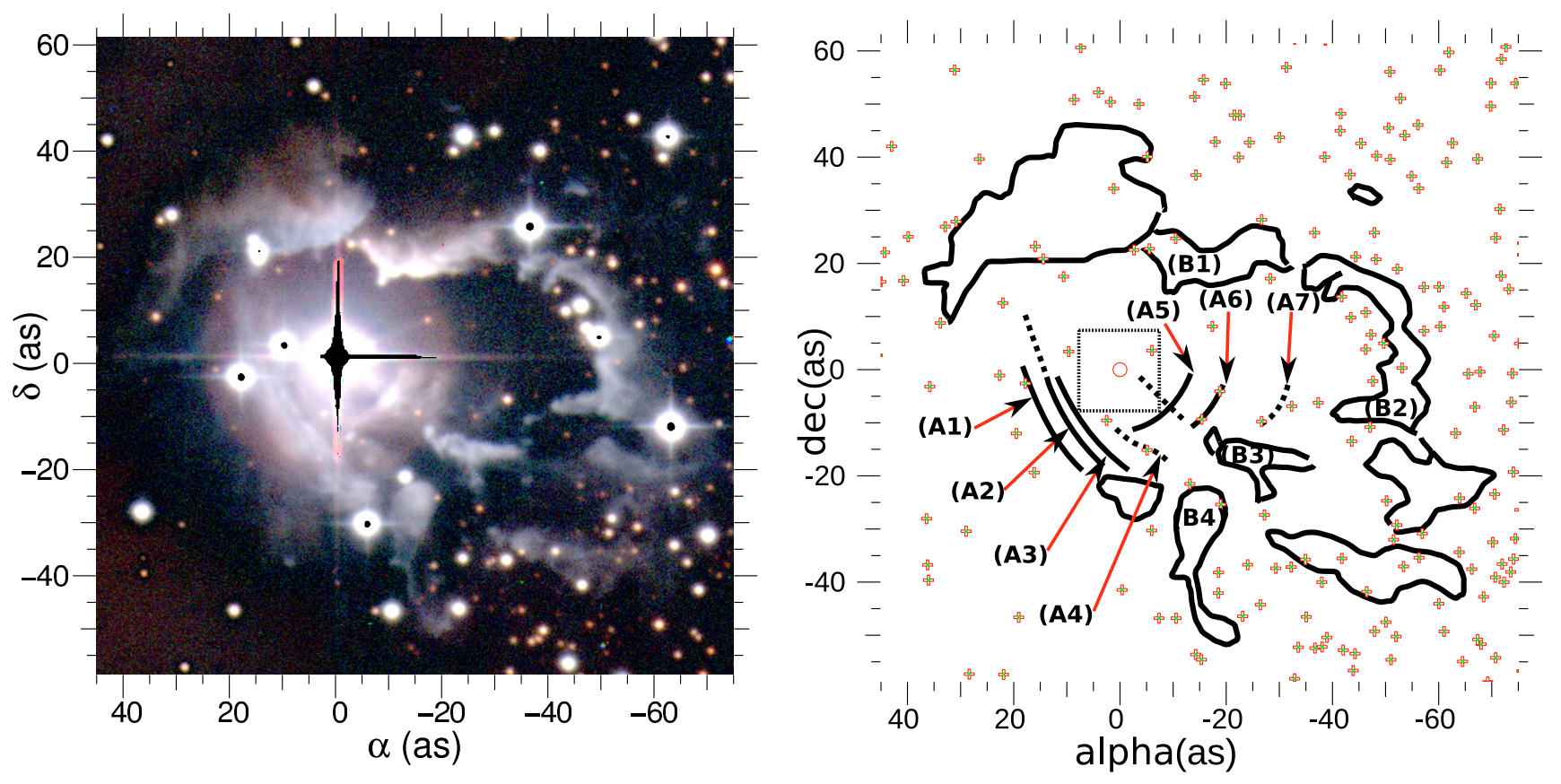

Fig. 6. Reflection nebula around HD 87643, as a composite of $R, V$ and $B$ filters (left), together with a sketch presenting the main structures (right). The saturated regions are masked by black zones in the image. This image is a small part of the $30^{\prime} \times 30^{\prime}$ field-of-view of the WFI observations. The sketch shows HD 87643 as a red circle, whereas other stars are marked as red crosses. The nebular contours are drawn as black lines and the prominent features are labelled (A1) to (A7) for arc-like structures and (B1) to (B4) for apparently blown-up nebular structures. Structures which are faint or uncertain are marked as dotted lines.

data. It is more difficult to derive an accurate flux ratio, given the complexity of the object. Here, we shall summarise our results, from the most constrained ones to the most speculative.

- The plane-of-sky separation of the components is $34 \pm$ 0.5 mas. At $1.5 \mathrm{kpc}$, it corresponds to a projected separation of $51 \pm 0.8 \mathrm{AU}$. As a comparison, the separation of the interacting components of $\beta$ Lyrae (Zhao et al. 2008) is of the order of $0.2 \mathrm{AU}$ (orbital period $12 \mathrm{~d}$ ), the separation of the $\gamma^{2}$ Velorum (Millour et al. 2007) colliding-wind components is of the order of $1.3 \mathrm{AU}$ (period $78 \mathrm{~d}$ ), and the approximate separation of the high-mass binary $\theta^{1}$ Ori C (Stahl et al. 2008; Patience et al. 2008; Kraus et al. 2007) is 15-20 AU (orbital period of between 20 and $30 \mathrm{yr}$ ).

- Therefore, the orbital period of HD87643 is large, approximately 20-50 years. In particular, no significant orbital motion is seen between our 2006 and 2008 data sets.

- The orbital plane might be seen at a high inclination (i.e. close to edge-on). Indeed, this is suggested by the high level of observed polarisation (Oudmaijer et al. 1998). The large-scale measurements also seem to imply a bipolar morphology (see Sect. 3.3). In addition, HD87643 shows photometric variability. The short-term variations (e.g. amplitude $\sim 0.5 \mathrm{mag}$, seen in the ASAS light curves and in Miroshnichenko 1998) are similar to Algol-like variability (Yudin \& Evans 1998) and are therefore probably a consequence of the time-variable absorption by the material passing through the line-of-sight. This is also in favour of a highly inclined (edge-on) disc-like structure.

- The orbit might be highly eccentric. This hypothesis is supported by the periodic structures seen in the large-scale nebula (see Sect. 3.3) that would suggest periodic eruptions. These eruptions would be a sign of close encounters of the binary components, while we observed a well-separated binary.

\subsection{A link to the larger-scale nebula}

Dust is found at large distances east and west of the nebula, with fluxes reaching $15 \mathrm{Jy}$ at $16.7 \mu \mathrm{m}$ (ISO/CAM1, offset of $25^{\prime \prime}$ from 
the star, aperture $20^{\prime \prime} \times 14^{\prime \prime}$ ), whereas no flux is detected in the north and south positions ${ }^{4}$. This points to a bipolar nebulosity in the east-west direction.

As mentioned by Surdej et al. (1981), the number of field stars decreases in the south-east direction. Also from this work (Sect. 2.3), the number of visible stars in the eastern part of the WFI image of the nebula is about 2 times less than in the western part. This may mean that the other side of a symmetrical bipolar nebula remains hidden in our WFI image.

Finally, we note that inferring the geometry of the nebula is potentially of great importance as this reflection nebula allows one to study the central star from different viewing angles, i.e probing different latitudes of the central star. The anisotropy of the star flux was already noted in Surdej et al. (1981) and Surdej \& Swings (1983).

Oudmaijer et al. (1998) and Baines et al. (2006) measured expansion velocities of $\approx 1000 \mathrm{~km} \mathrm{~s}^{-1}$. Therefore one can estimate an ejection time for the nebula: at $1.5 \mathrm{kpc}$ distance and taking a $50^{\prime \prime}$ extent, one gets $\approx 355 \mathrm{yr}$.

For the arc-like structures and the same value for the expansion speed and distance, we find the following values: $\approx 71 \mathrm{yr}$ for $\mathrm{A} 1, \approx 85 \mathrm{yr}$ for $\mathrm{A} 2, \approx 100 \mathrm{yr}$ for $\mathrm{A} 3$, and $\approx 128 \mathrm{yr}$ for $\mathrm{A} 4$. This first series gives ejection time intervals of $\approx 14, \approx 14$ and $\approx 28 \mathrm{yr}$, respectively, between two consecutive arcs. This might be the trace of a periodic ejection with a period of $\approx 14 \mathrm{yr}$ on the assumption that one arc (between A3 and A4) is not seen in our image. However, this periodicity is probably affected by a projection effect since the $v \sin i$ of the arcs is not known and they appear to be almost linear (instead of circular); hence, the $\approx 14 \mathrm{yr}$ periodicity is a lower limit. Concerning the second series of arcs, we find $\approx 85 \mathrm{yr}$ for $\mathrm{A} 5, \approx 135 \mathrm{yr}$ for $\mathrm{A} 6$, and $\approx 213 \mathrm{yr}$ for $\mathrm{A} 7$. In this case, the apparent ejection time intervals between two consecutive arcs are $\approx 50 \mathrm{yr}$ and $\approx 78 \mathrm{yr}$. Given that the arcs appear almost circular in the image, we can assume that the projection angle is close to $90^{\circ}$. Since we have only three arcs in this case (with one barely seen), we can only put an upper limit of $\approx 50 \mathrm{yr}$ on the periodicity of these ejections.

These broken structures suggest short, localised ejection that might coincide with short periastron passages of the companion, triggering violent mass-transfer between the components. Therefore, we tentatively infer limits between 14 and $50 \mathrm{yr}$ for the periodicity of the binary system. Monitoring the system at high angular resolution over a timescale of a few dozen years would most likely bring an unprecedented insight into this system.

\subsection{The nature of HD 87643}

Our interferometric measurements show a complex object composed of a partially resolved primary component, a compact secondary component, and a fully resolved component (i.e. extended, or nebular, emission). As shown in Table 3, their relative flux strongly varies between 1.6 and $13 \mu \mathrm{m}$.

The $L$-band NACO image of HD 87643 cannot be distinguished from the calibrator star, and there is very little emission at $1^{\prime \prime}-5^{\prime \prime}$ distance. In the $K$-band, except from the binary signature, no flux can be detected at a larger distance. Given the amount of dust in the system (McGregor et al. 1988), the compactness of the near-IR emissions indicate that most of it resides in a region smaller than $\approx 100$ mas. We may assume that the $K$-band flux comes entirely from the very central source, as

\footnotetext{
${ }_{4}$ These measurements can be found in the ISO database: http:// iso.esac.esa.int
}

Table 4. Estimated fluxes for each component from the model fitting of Sect. 2.1.2.

\begin{tabular}{lccc}
\hline \hline $\begin{array}{l}\text { Band or } \lambda \\
\text { (total value })\end{array}$ & $\begin{array}{c}\text { Primary } \\
\left(\mathrm{W} / \mathrm{m}^{2} / \mu \mathrm{m}\right)\end{array}$ & $\begin{array}{c}\text { Background } \\
\left(\mathrm{W} / \mathrm{m}^{2} / \mu \mathrm{m}\right)\end{array}$ & $\begin{array}{c}\text { Secondary } \\
\left(\mathrm{W} / \mathrm{m}^{2} / \mu \mathrm{m}\right)\end{array}$ \\
\hline$H(4.8 \mathrm{mag})$ & $8.5 \times 10^{-12}$ & $1.6 \times 10^{-12}$ & $3.7 \times 10^{-12}$ \\
$K(3.5 \mathrm{mag})$ & $1.2 \times 10^{-11}$ & $1.5 \times 10^{-12}$ & $3.1 \times 10^{-12}$ \\
$8.1 \mu \mathrm{m}(92 \mathrm{Jy})$ & $7.2 \times 10^{-13}$ & $2.9 \times 10^{-12}$ & $5.9 \times 10^{-13}$ \\
$10.5 \mu \mathrm{m}(146 \mathrm{Jy})$ & $3.5 \times 10^{-13}$ & $3.0 \times 10^{-12}$ & $6.0 \times 10^{-13}$ \\
$12.9 \mu \mathrm{m}(126 \mathrm{Jy})$ & $1.4 \times 10^{-13}$ & $1.7 \times 10^{-12}$ & $4.3 \times 10^{-12}$ \\
\hline
\end{tabular}

We used 2MASS magnitudes as the total magnitude in $H$ and $K$-band and the MIDI fluxes in $N$-band.

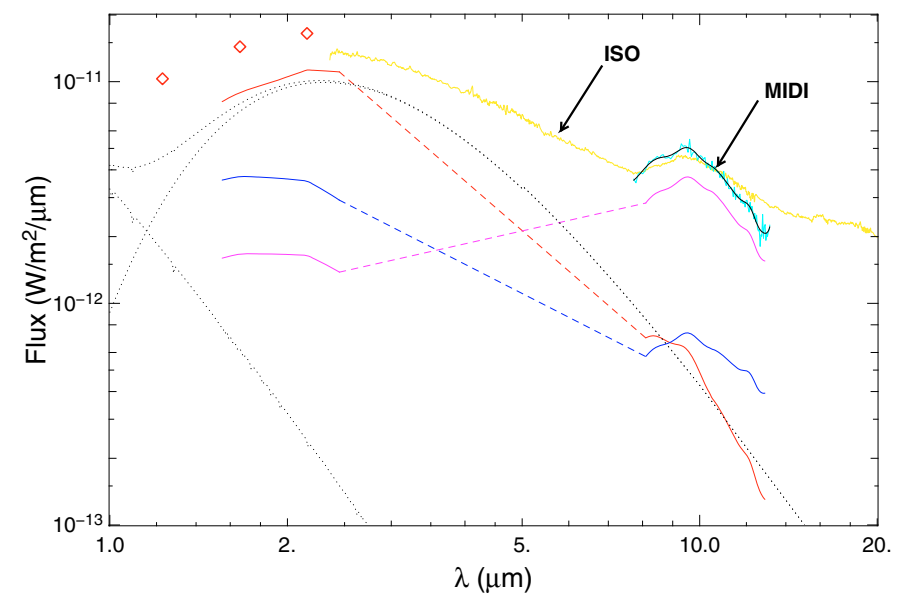

Fig. 7. A view of the (non-de-reddened) HD87643 SED with the extracted fluxes from our interferometric measurements. The southern component flux is shown in red (top curve at $2 \mu \mathrm{m}$ ), the northern component flux is shown in blue (middle curve at $2 \mu \mathrm{m}$ ), and the resolved background flux is shown in pink (bottom curve at $2 \mu \mathrm{m}$ ). The dotted lines correspond respectively to a Kurucz spectrum of a B2V star and to a black-body flux at $1300 \mathrm{~K}$, for comparison.

seen by NACO (i.e., it contains the central binary star plus the extended emission, as seen by AMBER). We assume the same for the $H$-band. Given these hypotheses, we can infer the absolute flux of each component using (for example) the 2MASS magnitudes in the $H$ and $K$-bands.

In the mid-infrared, the MIDI spectrum is close to the ISO one. Therefore, we can also assume that all the $N$-band flux originates from the MIDI field of view (i.e. $\sim 1^{\prime \prime}$ ) and infer the absolute fluxes of the components in the $N$-band. These fluxes $(N$ band) and magnitudes ( $H$ and $K$-bands) are presented in Table 4 and plotted in Fig. 7 as a function of the wavelength.

Even if the effect of reddening on the $H$ and $K$-band flux is not negligible, a significant reddening fraction comes from the circumstellar envelope. Therefore we performed this study on the original measurements, without de-reddening.

\subsubsection{A dust-enshrouded star in the south}

The flux of the envelope around the southern component, from the AMBER and MIDI data, can be qualitatively described by a $1300 \mathrm{~K}$ black body radiation (dotted line in Fig. 7). Its Gaussian $F W H M$, inferred from the model-fitting, is $\sim 4$ mas (i.e. $6 \mathrm{AU}$ at $1.5 \mathrm{kpc}$ ) in the near-infrared ( $H$ and $K$-bands), and is well constrained by the AMBER 2008 data. These suggest an extended dusty envelope around this component and clearly indicate that the dust must be very close to the sublimation limit. Thus, the 
$H$ and $K$-band emission should mainly originate from the inner radius of a dusty disc, encircling a viscous gas disc or a 2-component wind (Porter 2003). Estimating the envelope extension with a ring instead of a Gaussian would lead to a slightly smaller size. Thus, we can estimate that the inner radius of the dusty disc is of the order of $2.5-3 \mathrm{AU}$ (at $1.5 \mathrm{kpc}$ ).

\subsubsection{A puzzling dusty object in the north}

By contrast, the northern component is unresolved (size, or diameter, of the source $\leq 2$ mas, i.e. $\leq 3 \mathrm{AU}$ at $1.5 \mathrm{kpc}$ ). Moreover, its flux can be accounted for neither by a simple black-body at a constant temperature nor by free-free emission. The slope of the flux variation between the $H$ and $N$-bands suggests a range of temperatures for the dust (from at least $\approx 300 \mathrm{~K}$ to $\approx 1300 \mathrm{~K}$ ). No cold dust can survive so close ( $T \approx 300 \mathrm{~K}$ at a radius $\leq 1.5 \mathrm{AU}$ ) to a putative luminous hot star without effective screening of the stellar radiation.

\subsubsection{A cold dust circumbinary envelope}

The resolved component shows a large increase of flux between $H, K$, and $N$ and also carries most of the silicate emission (see Fig. 7). The binary system is therefore embedded within a large oxygen-rich, dusty envelope whose shape is not constrained by our data. This envelope must significantly contribute to the reddening of both components.

In conclusion, we propose the following picture of the system: two stars with dusty envelopes probably surrounded by a common dusty envelope. One source might be a giant or a supergiant hot star surrounded by a disc, in which we would mainly see the inner rim corresponding to the dust sublimation radius. The other source is surrounded by a compact dusty envelope, and it is either not a luminous hot star or it is heavily screened by very close circumstellar matter. In the $N$-band, the circumbinary envelope contribution is not greater than $\approx 1^{\prime \prime}$, since the MIDI (aperture $1.2^{\prime \prime}$ ) and ISO (aperture $22 \times 14^{\prime \prime}$ ) fluxes match very well, and is not smaller than $\approx 200$ mas, as our MIDI data indicates.

\section{Concluding remarks}

Our work presents new observations of HD87643, including very high angular resolution images. It completely changes the global picture of this still puzzling object:

- The binary nature of the system has been proved, with a projected physical separation of approximately 51 AU in 2008 at the adopted distance of $1.5 \mathrm{kpc}$. The orbital period is most likely several tens of years, and the large-scale nebula indicates a possible high eccentricity.

- The temperature of the southern component is compatible with an inner rim of a dusty disc. In the near-IR, we do not see the central star, but it might be a giant or a supergiant star.

- The northern component and its dusty environment are unresolved. The underlying star is unlikely to be a massive hot star, or is heavily screened by close-by circumstellar material, as cold dust $(T \approx 300 \mathrm{~K}$ ) exists closer than 1.5 AU from the star (if it lies at $1.5 \mathrm{kpc}$ ).

- The system is embedded in a dense, circumbinary, and dusty envelope, larger than 200 mas and smaller than $1^{\prime \prime}$.

High angular resolution observations were definitely vital in partly revealing the nature of this highly intriguing object.
Therefore, we call for future observations of this system, using both high spectral resolution spectroscopy and high angular resolution techniques, to place this interesting stellar system on evolutionary tracks and better understand its nature.

Acknowledgements. F. Millour and A. Meilland are funded by the Max-Planck Institut für Radioastronomie. M. Borges Fernandes works with financial support from the Centre National de la Recherche Scientifique (France). The research leading to these results has received funding from the European Community's Sixth Framework Programme through the Fizeau exchange visitors programme. This research has made use of services from the $\mathrm{CDS}^{5}$, from the Michelson Science Centre ${ }^{6}$, and from the Jean-Marie Mariotti Centre ${ }^{7}$ to prepare and interpret the observations. Most figures in this paper were produced with the scientific language yorick ${ }^{8}$. The authors thank K. Murakawa for fruitful discussions.

\section{References}

Allen, C. W. 1973, Astrophysical quantities (London, University of London: Athlone Press), 3rd ed.

Allen, D. A., \& Swings, J. P. 1976, A\&A, 47, 293

Baines, D., Oudmaijer, R. D., Porter, J. M., et al. 2006, MNRAS, 367, 737

Baron, F., \& Young, J. S. 2008, in SPIE Conf. Ser., 7013

Béchet, C., Tallon, M., Tallon-Bosc, I., et al. 2005, in SF2A-2005: Semaine de 1'Astrophysique Francaise, ed. F. Casoli, T. Contini, J. M. Hameury, \& L. Pagani, 271

Bertin, E., \& Arnouts, S. 1996, A\&AS, 117, 393

Bjorkman, J. E., \& Cassinelli, J. P. 1993, ApJ, 409, 429

Cidale, L., Zorec, J., \& Tringaniello, L. 2001, A\&A, 368, 160

Conti, P. S. 1997, in Luminous Blue Variables: Massive Stars in Transition, ed. A. Nota, \& H. Lamers, ASP Conf. Ser., 120, 161

de Freitas Pacheco, J. A., Gilra, D. P., \& Pottasch, S. R. 1982, A\&A, 108, 111

Domiciano de Souza, A., Driebe, T., Chesneau, O., et al. 2007, A\&A, 464, 81

Haniff, C. 2006, New Astron. Rev., 51, 583

Hanson, M. M., Conti, P. S., \& Rieke, M. J. 1996, ApJS, 107, 281

Hofmann, K.-H., \& Weigelt, G. 1993, A\&A, 278, 328

Kraus, M. 2006, A\&A, 456, 151

Kraus, M., \& Lamers, H. J. G. L. M. 2003, A\&A, 405, 165

Kraus, S., Balega, Y. Y., Berger, J.-P., et al. 2007, A\&A, 466, 649

Lamers, H. J. G., \& Pauldrach, A. W. A. 1991, A\&A, 244, L5

Lamers, H. J. G. L. M., Zickgraf, F.-J., de Winter, D., Houziaux, L., \& Zorec, J. 1998, A\&A, 340, 117

Leinert, C., Graser, U., Waters, L. B. F. M., et al. 2003, in SPIE Conf. Ser., ed. W. A. Traub, 893

Leinert, C., van Boekel, R., Waters, L. B. F. M., et al. 2004, A\&A, 423, 537

Lopes, D. F., Damineli Neto, A., \& de Freitas Pacheco, J. A. 1992, A\&A, 261, 482

McGregor, P. J., Hyland, A. R., \& Hillier, D. J. 1988, ApJ, 324, 1071

Millour, F., Tatulli, E., Chelli, A. E., et al. 2004, in SPIE Conf. Ser. 5491, ed. W. A. Traub., 1222

Millour, F., Petrov, R. G., Chesneau, O., et al. 2007, A\&A, 464, 107

Millour, F. 2008a, New Astron. Rev., 52, 177

Millour, F., Valat, B., Petrov, R. G., et al. 2008b, in SPIE Conf. Ser., 7013

Miroshnichenko, A. S. 1998, in B[e] stars, ed. A. M. Hubert, \& C. Jaschek, 145, Astrophys. Space Sci. Library, 233

Miroshnichenko, A. S., Levato, H., Bjorkman, K. S., et al. 2004, A\&A, 417, 731

Miroshnichenko, A. S., Bernabei, S., Polcaro, V. F., et al. 2006, in ASP Conf. Ser. 355, Stars with the B[e] Phenomenon, ed. M. Kraus \& A. S. Miroshnichenko, 347

Monnier, J. D. 2006, New Astron. Rev., 51, 604

Olnon, F. M., Raimond, E., Neugebauer, G., et al. 1986, A\&AS, 65, 607 Oudmaijer, R. D., Proga, D., Drew, J. E., et al. 1998, MNRAS, 300, 170

Patience, J., Zavala, R. T., Prato, L., et al. 2008, ApJ, 674, L97

Petrov, R. G., Malbet, F., Weigelt, G., et al. 2007, A\&A, 464, 1

Pojmański, G. 2009, in ASP Conf. Ser., ed. K. Z. Stanek, 52, ASP Conf. Ser., 403

Porter, J. M. 2003, A\&A, 398, 631

Ratzka, T., Leinert, C., Henning, T., et al. 2007, A\&A, 471, 173

Rousset, G., Lacombe, F., Puget, P., et al. 2003, in SPIE Conf. Ser. 4839, ed. P. L. Wizinowich, \& D. Bonaccini, 140

Rué, F., \& Bijaoui, A. 1997, Exper. Astron., 7, 129

Shore, S. N., Brown, D. N., Bopp, B. W., et al. 1990, ApJS, 73, 461

\footnotetext{
5 http://cdsweb.u-strasbg.fr

6 http://msc.caltech.edu

7 http://www. jmmc. fr

8 http://yorick. sourceforge.net
} 
Stahl, O., Wade, G., Petit, V., Stober, B., \& Schanne, L. 2008, A\&A, 487, 323 Stee, P., Meilland, A., Berger, D., et al. 2005, in The Nature and Evolution of Disks Around Hot Stars, ed. R. Ignace, \& K. G. Gayley, ASP Conf. Ser. 211, 337

Surdej, J., \& Swings, J. P. 1983, A\&A, 117, 359

Surdej, A., Surdej, J., Swings, J. P., et al. 1981, A\&A, 93, 285

Tatulli, E., Millour, F., Chelli, A., et al. 2007, A\&A, 464, 29

Thiébaut, E. 2008, in SPIE Conf. Ser., 7013

Valenti, J. A., Johns-Krull, C. M., \& Linsky, J. L. 2000, ApJS, 129, 399

van den Bergh, S. 1972, PASP, 84, 594
Voors, R. H. M. 1999, Ph.D. Thesis, Universiteit Utrecht, The Netherlands, Yudin, R. V., \& Evans, A. 1998, A\&AS, 131, 401

Zhao, M., Gies, D., Monnier, J. D., et al. 2008, ApJ, 684, L95

Zickgraf, F.-J. 2003, A\&A, 408, 257

Zickgraf, F.-J., Wolf, B., Stahl, O., Leitherer, C., \& Klare, G. 1985, A\&A, 143, 421

Zorec, J. 1998, in Astrophysics and Space Science Library 233, B[e] stars, ed. A. M. Hubert, \& C. Jaschek, 27

Zsargó, J., Hillier, D. J., \& Georgiev, L. N. 2008, A\&A, 478, 543 


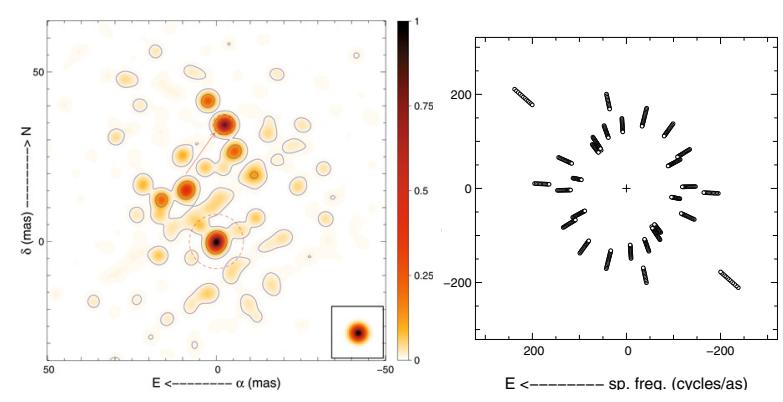

Fig. A.1. Removing low spatial frequencies to check whether the structures are reconstructed.

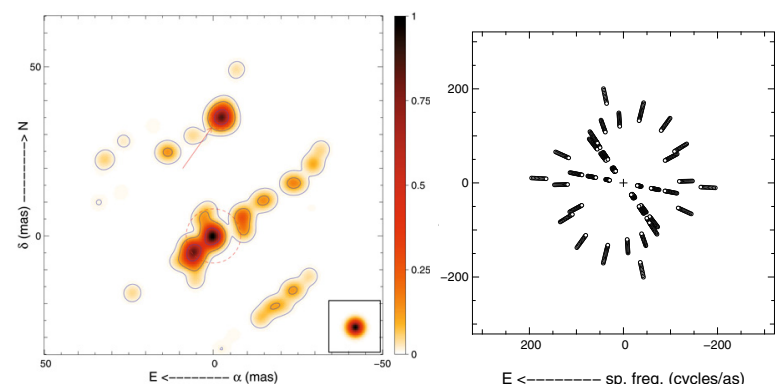

Fig. A.2. Removing high spatial frequencies to have a more symmetric UV coverage.

\section{Appendix A: MIRA imaging of HD87643: tests and reliability estimates}

This work made use of the MIRA software to reconstruct images from the sparsely sampled AMBER interferometric data. In this appendix, we present test results demonstrating which structures in the reconstructed images are reliable.

\section{A.1. Altering the UV coverage}

The idea here is to find out if the structures remain qualitatively the same in the final image when altering the resolution (removing the high spatial frequencies) or the low frequency components of the UV plane.

\section{A.1.1. Removing low spatial frequencies}

Removing low spatial frequencies reduces the field of view, and the MIRA image reconstruction software does not manage to overcome this difficulty (see Fig. A.1). Therefore, we conclude that the low spatial frequencies (i.e. the short baselines) are just as important as longer baselines to perform image reconstruction.

\section{A.1.2. Removing high spatial frequencies}

As a second test of the image reconstruction reliability, we cut all the high spatial frequencies in order to get a more symmetric UV coverage. The result is seen in Fig. A.2, together with the corresponding UV coverage. The striking point compared to the image presented in Fig. 2 is that all the structures seen in this new image are qualitatively the same as before. This ensures that the binarity and resolution of the southern components are not due to image reconstruction artifacts.

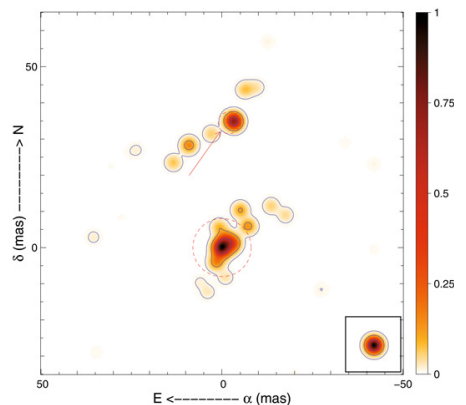

Fig. A.3. HD 87643 image reconstruction without the phase information. We had to rotate the image by $180^{\circ}$ to make it match the previous reconstructions.

\section{A.1.3. Imaging without phases}

Since the AMBER closure phases in our data-set were noisy ( $\sigma \approx 0.5-1$ radian), we wondered whether these phases were contributing significant information to the image reconstruction compared to squared visibilities alone.

We tried to reconstruct an image of HD87643 using the same AMBER data, except for the closure phases, and both the MIRA and BSMEM image reconstruction software. Indeed, they are able to cope with phase-less data, in this case making a phaseretrieval image reconstruction. In Fig. A.3 we show the MIRA reconstruction. We are able to reconstruct qualitatively the same image of HD87643 as in Fig. 2 without using the closure phase information.

We note, however, that we had to rotate the result to match the orientation of Fig. 2. This makes sense since only the phase information would be able to orient the system.

\section{A.2. Testing with other image reconstruction algorithms}

MIRA is only one example of an image reconstruction software package for optical interferometry. Other software packages exist, and we present here the tests we made using several of them.

\section{A.2.1. BSMEM}

BSMEM is based on the maximum entropy method (MEM) applied to bispectrum measurements (deduced from the squared visibilities and closure phase measurements). It works using an iterative algorithm, comparing the distance from the reconstructed image to the data and the "entropy" computed from the properties of the image itself (Baron \& Young 2008). We applied BSMEM to the AMBER data. The result can be seen in Fig. A.4. We find the same structures as for the MIRA reconstruction process.

\section{A.2.2. Building-block method}

The building-block method (Hofmann \& Weigelt 1993) was developed to reconstruct diffraction-limited images from the bispectrum of the object obtained with bispectrum speckle interferometry and long-baseline interferometry. Since the intensity distribution of an object can be described as a sum of many small components, the building-block algorithm iteratively reconstructs images by adding building blocks (e.g. $\delta$-functions). The initial model image may simply consist of a single $\delta$ peak. Within each iteration step, the next building block is positioned at the particular coordinate, which leads to a new model 
F. Millour et al.: A binary engine fuelling HD 87643's complex circumstellar environment, Online Material p 2

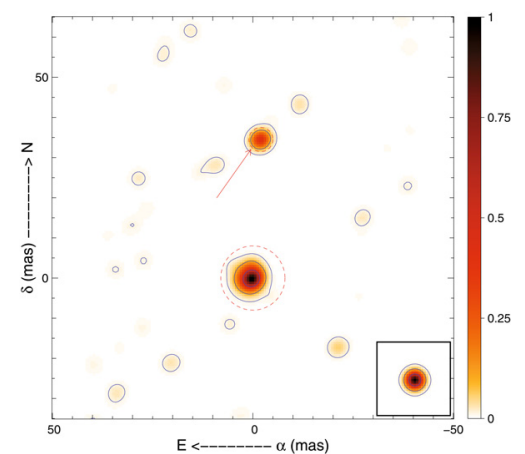

Fig. A.4. Image reconstruction of HD87643 using the BSMEM software.

image that minimises the deviations $\left(\chi^{2}\right)$ between the model bispectrum and the measured object bispectrum elements. An approximation of the $\chi^{2}$-function was derived which allows fast calculation of a large number of iteration steps. Adding both positive and negative building blocks, taking into account the positivity constraint, and adding more than one building block per iteration step improves the resulting reconstruction and the convergence of the algorithm. The final image is obtained by convolving the building-block reconstruction with a beam matching the maximum angular resolution of the interferometer. We applied this method to the AMBER data, and the resulting image (Fig. A.5) shows many similarities with the MIRA one, including a resolved southern component in the $K$-band.

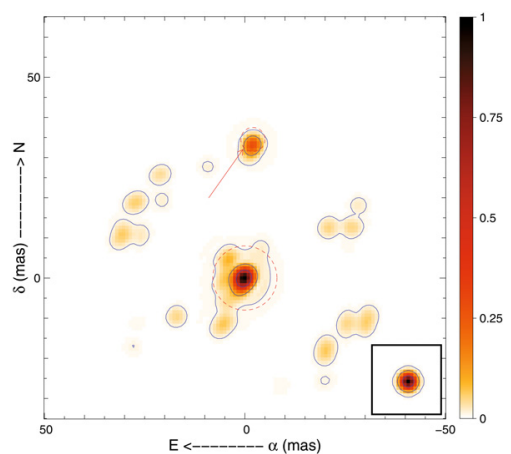

Fig. A.5. Image reconstruction of HD87643 using the building-block software.

\section{A.3. Conclusion}

The tests presented above show that the binary and resolved southern component are structures that can be trusted in the images. All other structures (possible elongation of the southern component, inclined large "stripes" in the images) are artifacts from the image reconstruction process, which may indicate that the image has some additional flux, not constrained by the observations (fully resolved background). 